\title{
Real Life Experiments on Interface Design
}

\author{
Luciane Maria Fadel \\ Federal University of Paraná
}

\begin{abstract}
This paper focuses on the choice of methods used to explore the interface design with the aim of increasing the number of interactions and enhancing social presence in an online environment. The research about interface design comprised four fieldbased experiments. The experiments focused on the design of different social facilities, but shared many similarities in terms of data collection and analysis. In addition, this paper describes how the online courses were designed and explains the experimental procedures common to all four experiments. Moreover, this paper discusses some of the challenges involved in conducting 'real' life experiments on interfaces in an online environment. Some of these challenges, such as students dropping of the courses and participants meeting in other face-to-face courses, do not have simple solutions. Others, such as technical problems should be carefully considered before the experiment starts. Furthermore, 'real' life experiment implies in less control over the variables. For this reason, the combination of qualitative and quantitative methods of analysis should be considered.
\end{abstract}

\section{Introduction}

Research into the interface for online learning environments is still in its infancy, and as technology, storage and retrieval capacity expand there will likely be numerous improvements enabling more flexible information design. At present however, there is scope for exploring the impact of interface design on social interaction, with a view to understanding how the visibility of interaction features, and different forms of presenting social information and feedback, can encourage students to interact and thus enhance social presence. This paper discusses the planning of experiments that evaluate the relationship between the interface design and students' interaction and perceptions. More specifically, this paper discusses some issues about conducting empirical tests and gathering data from 'real' life situation to provide research findings that address how the interface design supports social interaction and enhances social presence in an online learning environment.

\section{Field experiments}

The research adopted field experiments in order to achieve an authentic activity [2]. To be authentic the students should have genuine reasons to be connected to the learning activity. In addition, the students should attain meaningful goals and be intellectually and emotionally engaged in the tasks they carry out. In this case, the activities should promote collaboration and be arranged so that the students could attain goals which are difficult to reach alone. The tutors were instructed, therefore, to develop such activities and the environments created for the experiments described in this paper, should give means of collaboration, such as synchronous and asynchronous communication features. In addition, the learning environment must be able to support the learning experience in an authentic context. In the experiments described in this paper, the students were enrolled in a mandatory course offered by the Universidade do Oeste de Santa Catarina Videira (UNOESC VDA) in Brazil. The online courses were designed as a relevant online learning activity placed within the larger context of the goals of the University.

The intervention was restricted to the mediating tool of the activity, which was the online learning environment, and the associated synchronous and asynchronous communication tools. The experimental design preserved the authenticity of the actions performed within the online learning environment while allowing the tool to be investigated.

However, the design of a field experiment needs to deal with unpredictability. This could be generated by technical problems, students dropping out of courses, and the educational policies of the University. For example at UNOESC VDA, students' final grades must have a written text as part as their assessment, which implies a face-to-face meeting. Students also attended other face-to-face courses together. These factors could have implications for the perceived social presence. Another problem faced during the experiments was the absence during weekends of a technician at the University. This caused delays in restarting the server after a blackout or interruption of the services. 
In addition, the experimenter was based in UK while the experiments were carried out in Brazil. This means that no student behaviour could be observed. Nor, interviews could be carried out without mediation.

Next section describes the design of the online learning environment, the participants, and data collection.

\section{Online learning environment}

The community of inquiry framework explained in Fadel [4], was used to design the courses. In addition, paced collaborative learning was adopted which focuses the learning experience on the student-student interactions. This means that the courses were created to support adequate levels of teacher presence, cognitive presence and social presence in the context of student-student interactions.

The strategy to support teacher presence was asking the tutor to challenge the students' thinking and question the students' assumptions and point out other views to broaden the students' perspectives but mainly the tutor was expected to instigate the discussion among the students. In addition, the tutor should create a positive, supportive learning climate, by acknowledging student success, interacting in a respectful manner and being astute to arising problems. From the perspective of student-student interaction, the teacher presence should not be central. The teacher would guide the learning experience.

To create levels of cognitive presence students were provided opportunities to not only discuss what they had learned, but also apply what they had learned. Therefore, the learning experience was based on reasoned discourse, interactive participation and activities that were meaningful and challenging. Strategies to support social presence were the focus of the research. These strategies were concerned with the relationship between the interface design and student-student interactions. These interactions were supported by asynchronous and synchronous devices such as, forum, web mail, chat and instant mailing. The interface design for the online environments used in the experiments was informed by theories about perception and graphic design.

The first experiment used the interface design that was already in use at UNOESC VDA. Although the graphic interface was not modified some mechanisms were implemented to record the data exchange among students.

The second experiment was designed to compare the interface used in experiment 1 , referred to as the original interface, and another interface based on visibility, referred to as the visibility-based interface. The development of the visibility-based interface was informed by theory about attracting users' attention and used the principles of simplicity, emphasis, organisation and regularity.

The third experiment was designed to compare two interfaces presenting social information: one based on text, namely the text-based interface; and another based on images, animation and text, referred to as the visual-based interface. The textbased interface was practically the same as the visibility-based interface used in experiment 2 .

The fourth experiment had the same overall design as experiment 3 for both interfaces and system feedback added to the interfaces. This experiment was designed to compare a text-based interface and an animation-based interface to alert students to incoming messages.

Piloting was used in experiments 3 and 4 and served to evaluate different aspects of the graphic interface design and database design.

\section{Pilot study}

The process of piloting was an essential step in the iterative process of designing the experiments. A small sample of people was invited to pilot the interface and a group of students were invited to pilot the course.

\subsection{Piloting the interface}

The design of the visual-based interface (experiment 3) may have added a level of complexity to its use. Thus, before starting the main study it was necessary to test whether the students would be able to find information, and to understand the structure of the interface and how to interact with it. In other words, it was necessary to test if the visual-based interface was easy to use. Therefore, the pilot study was designed to determine ease of use, and preferences for the text- and visual-based interfaces. Eight students were recruited. The sample consisted of five women and three men, all Graphic Design undergraduate students.

Data collected during this phase of the experiment included both observational and subjective reports elicited by a series of open-ended questions. The observational reports quantified the time taken and whether or not the task set was successfully completed. In addition, comments made by the participants during the pilot study were recorded to determine any difficulties and their patterns of use.

Prior to starting the pilot study each participant was provided with a description of the aims and procedure of the pilot study. In addition, the participants were asked to sign a consent form as part of the University of Reading's Ethics and Research Committee procedure.

Each participant in the pilot study was then instructed to complete a series of 12 tasks, grouped into the following categories: 
- Persistent social information: the tasks in this category were related to information about the other students, such as hobbies and contact numbers.

- Transient social information: this category was related to information about online users, such as user name, connection duration and location.

- Communication: the tasks for this category involved posting messages at forum or web mail.

- General use: this category was related to information about the content of the week's topic and agenda.

Three separate tasks were scheduled for each category. In the pilot study each participant used both interfaces. Two sets of tasks were undertaken using one interface and the other two sets using the other interface. In order to avoid the effects of familiarity with task order, the order of the sets of tasks was reversed for every participant. Changing the order in this way limited the effect of familiarity with the operation and organisation of the interfaces for participants and also limited the boredom participants might experience. The 4 sets of tasks were paired with the two interfaces in 8 possible combinations.

Each participant received a task sheet with the sequence of tasks. Some tasks involved other online users. Therefore, some students were simulated to be online. Once the pilot study had commenced, the time taken by each participant for the allotted tasks was recorded using a stopwatch. Additionally, during the pilot study, any comments made by the participants were recorded for subsequent review.

\subsection{Piloting the course}

An online course was needed that would enable participants to spend time in the online environment and interact with others. The course of Programming Languages was chosen because it started before the other courses, thus enabling time for adjustments in the environment if needed. The course of Programming Languages is a mandatory course offered to $3^{\text {rd }}$ year Computer Science students at the UNOESC VDA.

The pilot was intended to test the method and identify possible flaws in the experiment before the main study. Piloting the course would also permit testing that all necessary data had been recorded, especially from the visual-based interface. Data recorded from the text-based interface had been tested during experiment 2.

Twelve students were recruited for the pilot. All were male. Participants were assigned such that equal numbers used the text-based interface and the visual-based interface.
Data collection. Data was collected using three questionnaires and system logs. The questionnaires collected demographic data, perceptions of social presence and judgements of satisfaction. During the pilot study the wording and clarity of items were tested. This was done through informal interviews using the chat facility with 4 participants. All reported that the items were easy to understand. Thus, no modifications in the questionnaires were implemented. The questionnaires used in the pilot study were detailed in Section 7.2.

The first questionnaire contained demographic questions and was completed before the beginning of the course.

The second questionnaire consisted of 8 Likert-scale items aimed to find out the degree of social presence sensed by the students by investigating their perceptions about group belonging and visual awareness.

The third questionnaire consisted of 7 Likert-scale items and one open-ended question designed to assess students' satisfaction with the interface.

Procedure. The course was organised into eighteen weeks. On Tuesdays participants met in a chat session to discuss the weekly topic. The completed tasks were sent to the tutor using web mail. The chat sessions were scheduled for Tuesday evenings and lasted from two to three hours depending on students' needs.

The online meetings occurred during weeks 2 to 6 and 8 to 13, giving a total of 9 weeks of online meetings.

\section{Participants}

The participants of all experiments were undergraduate students at UNOESC VDA. There are several reasons for this:

- The tutor was willing to take part;

- There was usually a sufficient number of students;

- Since the students were enrolled on a course, the use of the system could be evaluated in the application domain rather than in a laboratory.

- The experimental approach adopted by the research established one group of participants for experiment 1 ; and a control and an experimental group for experiments 2, 3 and 4.

Participants were assigned to use one of the two interfaces in experiments 2, 3 and 4, such that equal numbers of participants used one or the other interface. The criteria used to assign the students to groups were age, gender and the report from the tutor about each student's previous performance in another course. The aim of assigning students to 
groups was to ensure that, as far as possible, both groups were equivalent in terms of these factors (age, gender and level of performance).

\section{Ethical issues}

All the participants in the experiments were invited, and consented to participating. Asking students to participate in experiments as a condition of passing a course is a practice that is considered acceptable, provided that no undue coercion was presented, and that the procedures provided an educational benefit to the participants [3]. In addition, the participation of students in online course experiments is crucial because they represent the class of users for whom the environment was created.

Prior to running the experiments it was necessary to submit details of the experimental design and procedure for clearance from the University of Reading's Ethics and Research Committee procedure.

All the participants were asked to sign a consent form as part of the University of Reading's Ethics and Research Committee procedure.

\section{Data collection}

The broad purpose of the research was to investigate the impact of the interface design on the number of interactions, the perceived social presence and satisfaction. For each experiment a specific aspect of interface design was chosen for investigation, for example, emphasis on interaction facilities. This independent variable was then manipulated to create different interfaces for comparison, for example by including a large area for presenting forum messages. The dependent variables were chosen by defining how the responses of interest were to be measured. For instance, the perceived social presence was investigated by a number of constructs seen as indicators of social presence, for example, the degree to which students perceived themselves to be part of the group (group belonging).

The research considered both subjective and objective responses, therefore it used data gathered through questionnaires, as well as participants' grades and system logs. In addition, debriefing sessions were carried out with the tutor using chat facility. During these sessions the tutor reported how the students were behaving, his impression about the course and any difficulty he could be facing. These sessions were informal and held at least twice during the course (one after 5 weeks of the beginning of the course and one at the end of the course). The informality of these sessions enabled the tutor to focus on those aspects of greatest relevance to him, yielding richer and more varied data. Indeed, these sessions allowed the gathering of some very interesting information about the complexity and richness of the interactions that occurs in a field experiment. For example, during experiment 2 the tutor reported that those students who had higher grades did not stay connected in chat long. These observations were considered in the analysis and design of each experiment.

\subsection{System logs}

System log data is a step-by-step recording of student interaction with the online learning environment and other students. The level of detail recorded was determined by what was chosen to be evaluated in each experiment and included mouse clicks, menu calls, and all messages sent. Because the aspects to be evaluated could vary from one experiment to the other, the system log was conceived to record as many data as possible.

The system log recorded data about the date and time that the students logged into the environment, and each page visited. Other data such as the content of the messages, sender, and receiver, were recorded in the Structured Query Language (SQL) database. For example, the forum service recorded the content, the title, sender and time/date of the message posted.

Using these logs it is possible to know the number of interactions, number of logs, time spent on each page and the content of messages for each student. Access to the content of the database was restricted to the experimenter.

\subsection{Questionnaires}

At least two questionnaires were used in each experiment to gather data about students' demographics, perceived social presence, and satisfaction.

Satisfaction and social presence in the questionnaires were measured by different indicators. A number of questionnaire items were associated with each indicator. Some of these items were based on previously published research, while others were developed iteratively during the course of the research by piloting the questionnaire and analysing data from previous experiments.

7.2.1. Demographic questionnaire. The first questionnaire contained demographic questions and was completed one week before the course started. This questionnaire included questions about the participant's gender, age, number of online experiences and the technical means of accessing the Internet. The results of this questionnaire were used to assign the students to each group in experiments 2 , 3 and 4 . In addition, the results were used to explore possible problems students reported with their prior 
experience of the Internet and technical means of accessing the Internet.

7.2.2. Measures of social presence. There is no widely accepted measure of social presence. Short, Williams, and Christie [9] used semantic differential scales to assess the social and emotional capabilities of the medium, i.e., insensitive-sensitive, cold-warm, impersonal-personal, and unsociable-sociable. Gunawardena and Zittle [6] developed a scale that consisted of 14 Likert-scale items addressing the social presence of a computer-mediated conferencing environment, particularly the concept of immediacy. Rourke, Anderson, Garrison and Archer [8] developed a template for analysing and coding transcripts from a computer conference, using the expression of emotions, open communication, and group cohesion as the categories that indicate elements of social presence [5].

$\mathrm{Tu}[10]$ asserts that social presence is a complicated construct and involves privacy, social relationships, communication styles, the nature of the task, feedback and immediacy, among other items. He developed a 42-item questionnaire that identified social context, online communication, and interactivity as factors that comprise social presence [10].

Biocca, Burgoon, Harms and Stoner [1] argued that researchers have developed measurements that are specifically suited to the technology they are studying. That means that the items and measures constructed from them cannot be generalised to use in other media. Thus, they have argued for a clarification of the definition of social presence, they also argued for a robust and detailed measure of social presence, which is beyond the scope of this paper.

The approach taken in this paper was to iteratively develop the items of the questionnaire, to assess the perception of social presence based on previously published research, piloting the questionnaire and analysing data from previous experiments. The aim was to design a concise questionnaire which assessed the students' perception of the presence of others in an online learning environment where the presence of others was supported by the interface.

7.2.3. Social presence questionnaire. The second questionnaire consisted of Likert-scale items and/or open-ended questions designed to assess social presence sensed by the students, investigating their perceptions about feedback, group belonging, and social information awareness.

The questionnaire created for experiments 1 and 2 was used to gather information concerning students' responses to the feedback they received from other students and immediacy based on the social presence questionnaire created by Gunawardena and Zittle [6]. For experiments 1 and 2, 10 Likert-scale items were used to assess each student's responses to the degree of social presence they perceived. The responses to this variable were elicited by a set of statements with which students were asked to agree or disagree.

The overall measure for each of these two responses (feedback and immediacy) was constructed from $n$ items, three items in the first response (feedback) and seven in the second response (immediacy) totalling 10, as detailed below.

Feedback from others: the extent to which students experienced that they received a response from others $(n=3)$

1. I worked in groups to do the tasks.

2. Other participants commented on my messages in the forum.

3. I didn't receive answers to my doubts and queries.

Immediacy: the extent to which the others are perceived as being psychologically close $(n=7)$

1. An online education course is an excellent medium to exchange ideas, chat and make friends.

2. Messages on this course were impersonal.

3. I didn't feel part of a virtual community.

4. My point of view was understood by other participants.

5. I felt comfortable interacting with other participants.

6. I felt lonely during this course.

7. I would like to participate in another distance learning course.

In experiment 3 , the questionnaire about social presence consisted of 8 Likert-scale items investigating their perceptions about group belonging and visual awareness. This questionnaire was the result of an iterative process. In this process, the questionnaire about social presence used in the previous experiment was analysed and the items were regrouped or modified so that the answer could reflect the students' perception of the virtual users as real beings. In addition, the indicators pointed out by Garrison, Anderson and Archer [5] were considered. Experiment 3 focused on enhancing the presence of others, an aspect of the group cohesion indicator. The questionnaire therefore included items about group belonging and social information awareness as detailed below.

Group belonging: the feeling of participating in a group $(n=5)$

1. I did not feel part of a group.

2. I felt comfortable interacting with other participants.

3. I felt lonely during this course.

4. I received feedback. 
5. The messages I received were addressed to everyone (impersonal).

Social information awareness: perceiving the others $(n=3)$

1. I perceived the presence of other students.

2. I did not know what the others were doing.

3. I noticed for how long the others were connected.

As with the other questions, these 8 items used to assess each student's perception of their perceived social presence were a set of statements with which students were asked to agree or disagree. The responses used a 5-point Likert-scale.

For experiment 4, wording modifications were carried out after piloting the questionnaire. The context in which the students should report their answer was added. For example, item 1 was 'I did not feel part of a group'. This item was modified to 'I did not feel part of a group when I was online'. In addition, the Likert-scale was stated using the meaning instead of the number. Thus, instead of ' 1 ' this option was declared as 'strongly disagree'. These modifications were also implemented in the satisfaction questionnaire.

7.2.4. Satisfaction questionnaire. The third questionnaire aimed to find out students' satisfaction with the interface. The questions were designed to assess students' perceptions about their interactions with other students and about their user experience. This questionnaire also consisted of Likert-scale and/or open-ended questions items.

The first version of the questionnaire about satisfaction was created for experiment 2. The questions were designed to assess students' perceptions of satisfaction with the course as detailed below.

1. Did you like the course? Explain.

2. What didn't you like in this course? Explain.

3. Which activity (chat, forum, Web mail or content pages) did you like the most? Explain.

4. How was your interaction with the tutor?

5. What are your comments about studentstudent interactions?

6. Would you enrol in another online education course? Explain.

7. Would you like to suggest any changes to the interface?

8. Any other comments?

Because the questionnaire's items were openended it was not possible to apply statistics to the results. Statistics would allow verifying the correlation between satisfaction and other variables, such as visibility. Thus a new version, based on
Likert-scale items was created for experiment 3. This version consisted of 7 Likert-scale items and one open-ended question designed to assess students' satisfaction with the interface. The first three items were designed to assess students' perceptions about their interactions with other students. The other four items were designed to estimate their user experience as detailed below.

Perceptions about their interactions: the extent to which the students liked the interface as a mean to interact $(n=3)$

1. I found no means at the interface to get in touch with the other students.

2. I found means at the interface to get in touch with the tutor.

3. I did not feel encouraged to post.

User experience: the extent to which the students liked the online course $(n=4)$

1. I would like to participate in another distance learning course.

2. I was not able to learn from the course.

3. I did not like the course.

4. I liked the interface.

Open-ended question: an attempt to find any other information relevant to the students' experience $(n=1)$

1. Which activity, Chat, Forum, Web mail did you like the most? Explain.

In both questionnaires about social presence and satisfaction the wording of statements varied so that agreement might indicate either a strong or a weak sense of social presence or satisfaction respectively. This was done to try to ensure that each question was read, rather than the same response ticked for each item.

The items in the questionnaires with an asterisk

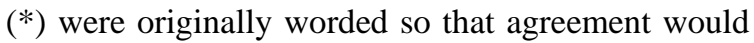
correspond to a weak perception of satisfaction or social presence. However, in order to collate the data, the responses were reverse coded. Taking the first question of the social presence questionnaire in experiment 1 as an example, the original was written as 'Messages on this course were impersonal', and its mean was 4.1, i.e. slight agreement. When reverse coded the question was written as a positive statement and its score was reversed.

The questionnaires about social presence and satisfaction were completed at the end of each course. The next section describes how the experiments were conducted and how the online courses were organised. 


\section{Procedures}

\subsection{Training}

In week one of each experiment, an introductory lecture and training about how to use the online learning environments were carried out before the experiment proper. The tutor explained to each group separately what they were supposed to do, how they should study, how they would be assessed. The lecture lasted approximately one hour and introduced the environments and their use. The training phase was carried out after the lecture. This phase lasted approximately one hour and was run informally to allow the students to ask questions and overcome any technical problems in using the environment.

\subsection{Conducting the experiment}

The courses were organised into fifteen to nineteen weeks depending on the content and school calendar. The courses were structured around reading and weekly discussions. In addition, practical tasks were required which were designed to get students to use their knowledge of theory. The completed tasks were sent to the tutor using web mail or using their university email when an attachment was necessary. Each week a different topic was presented to the students using the online learning environment. The students had a week to read the material and prepare the tasks. Once a week they met in a chat session to discuss the topic.

A timetable was given describing what event would occur, e.g., face-to-face or online meeting, and when they would occur. During the face-to-face meetings the students were given further explanations about the content. The tutor also gave feedback about how well the students were doing.

Email, forum and chat were the interaction features that students could use to communicate with each other. The only online scheduled discussions with the tutor were chat. The students were encouraged to post as many messages as they wanted in the forum, to email and to use chat to talk to their fellow students at any time.

In the experiment 2, 3 and 4 both groups used the chat room outside the online course environment because the students reported that chat in the environment was too slow. This technical problem was due to the fact that the chat page was constructed using HTML technology and because of the server configuration. This chat room did not have restricted access, which allowed people other than the students to participate in the chat session. Therefore, the messages exchanged among these non-students and the students were discarded. This chat room supported more than 20 users logged at the same time and it is faster than the chat room offered in the system.
Students were given responsibility for establishing their time and place of study. This flexibility allowed the students to manage times and places best suited to them.

\section{Results}

This section covers the methods used to analyse the data gathered in the four experiments. As mentioned earlier, the data included subjective questionnaires as well as objective system log data. Statistics were used to analyse the results when the number of participants were sufficient. Otherwise, the analyses were descriptive.

To test for normality using a sample size of more than or equal to 20 the Ryan-Joiner test (similar to the Shapiro-Wilk test) was used. In the other cases (sample size less than 20) the histogram analysis was used.

To test the hypotheses concerning relationships between variables a linear regression model was used [7]. A scatter plot was drawn for those variables which presented a strong relationship, for example, Figure 1 shows a scatter diagram showing the number of postings in forum plotted against social presence where the dashed lines represent the means. This diagram shows a strong positive correlation between the number of messages posted to the forum and the perceived social presence in experiment 2 .

When normality was verified the t-test was used to assess whether the means of the two groups were statistically different from each other. To test the significance, the risk level was set at .05 . In the other cases the Wilcoxon's Sum Rank test was used. The ttest is used to test the significance of the relationship between the degree of social presence and the student's performance.

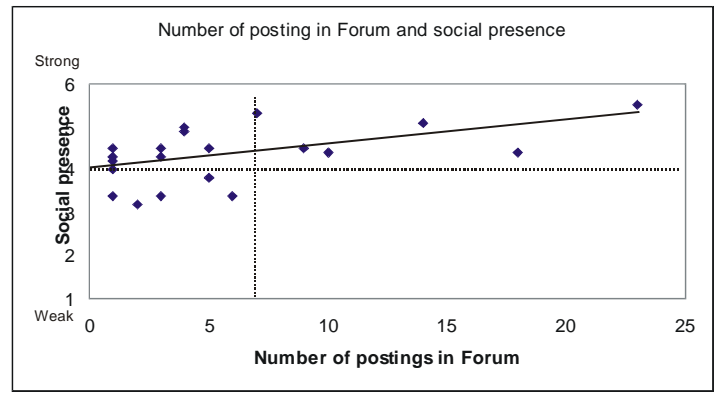

Figure 1. Number of posting in Forum and social presence

Wilcoxon's Sum-Rank test is used to determine whether the two groups differ in relation to the number of total messages posted and chat messages in experiments 3 and 4. Wilcoxon's Sum-Rank test is also used to determine whether the two groups differ in relation to the perceived social presence and in relation to satisfaction with the interface. 
In addition, the chi-square test was used to establish the significance of the difference between the two groups when there was a low participation of the students from one group. Therefore, chi-square was used to determine whether the two groups differ in relation to the number of messages posted at forum, web mail and instant mail features in experiments 3 and 4 .

In addition to their means, the perceived social presence and satisfaction were also reported by their frequency of responses. In order to calculate chisquare in experiments 3 and 4 , the frequencies of responses to 'strongly agree' and 'agree' were combined to form a single frequency. The same was done for the opposite end of the scale ('strongly disagree' and 'disagree'). This was done to increase the expected frequency, as the number of responses was low. However, some of the expected frequencies were still below 5 and chi-square tests could not be carried out.

If the students did not complete the questionnaires their result were registered as 'no data' in the corresponding tables. This happened with 6 students during experiment 3.

SQL was used for accessing and manipulating the data recorded in the system log. With SQL, the database was queried and a result set returned.

\section{Conclusion}

At present, online learning environments are used to deliver and manage the online education process. However, they have the potential to extend beyond their present usage to address the practical social interaction needs of geographically dispersed students. A chief attraction of the online learning environment lies in its ability to provide students with freedom of time and space. The challenge for designing such environments is to support groups of people to collaborate and interact socially in an efficient and enjoyable way, while permitting maximum student freedom.

One key barrier to effective social interaction in current online learning environments is the lack of social presence as compared to face-to-face communication. However, enhancing the perceived social presence involves significant challenges. Compared to face-to-face communication, online learning requires a balance between the distraction caused by too much information and the attention required to read the content. Social presence must therefore be traded off against the need to support focused attention. In terms of presentation, the tension between the use of text alone and images, animation and text underlines the difficulty for portraying information. Text alone would entail an unacceptable monotone. On the other hand, images, animation and text, whilst breaking the monotony, can add complexity to the information and may not be desirable in a medium where the students must have control over the process.

Given these constraints, the approach taken in this research was to explore the design of the social facilities that support social presence. The overarching goal was to investigate whether placing an emphasis on interaction facilities, incorporating text, images and animation, could affect students' experiences when interacting with each other. The theoretical foundation of social presence and the process of enhancing social presence was analysed through the literature review. Social presence was considered in terms of three categories of indicators determined by Garrison, Anderson and Archer [5]. These categories are the expression of emotions, group cohesion and open communication. Therefore, three variables that might affect these categories of indicators were chosen: participation, the presence of others and system feedback.

In addition, the relevant factors that influence the interface design enhancing social presence were identified and contributions to the practical realisation and evaluation of these factors were made. In this regard, an interface interaction model was developed. This interface interaction model proposed a set of key interface features that are essential to build online learning applications with adequate support for social interaction. These features are:

- The visibility of the interaction facilities;

- The use of images, animation and text to present the presence of others, and;

- The use of animation to present system feedback.

To validate the interface interaction model, four experiments have been carried out under real-life conditions in the context of online learning. The four experiments were conducted investigating the relationship between the number of interactions and social presence, and these three features of the design of social facilities that support social presence.

This research measured objective and subjective responses, combining student questionnaires with system logs and final grades. This research was not concerned, however, with the techniques involved in creating interfaces.

This paper explores the methods for conducting empirical tests and gathering data from 'real' life situation to provide research findings that address how the interface design supports social interaction and enhances social presence.

Real life experiments had impact on the results, for example, there was no statistical difference between the perceived social presence reported for both groups. The 'real-life' relationships, i.e., other face-to-face courses that the students were enrolled in, might have affected the perception of social presence. Neither is there a statistical difference in 
satisfaction between the groups. This finding might be explained because both interfaces corresponded to the expectations of the students.

In addition, this paper discussed some of the challenges involved in conducting 'real' life experiments on interfaces in an online environment. Some of these, such as students dropping of the courses and participants meeting in other face-toface courses, do not have simple solutions. Others, such as technical problems should be carefully considered before the experiment starts. Furthermore, 'real' life experiment implies in less control over the variables. For this reason, the combination of qualitative and quantitative methods of analysis should be considered.

Other contribution of this paper was the development and gradual refinement of questionnaire items relating to a selection of social responses including feedback, group belonging, and visual awareness. The measurements evolved through the course of the research. Their development was greatly aided by the use of qualitative analysis on the first questionnaire from the first experiment. For example, the two indicators used in the first version of the social presence questionnaire (feedback from others and immediacy) were substituted in the second version (group belonging and visual awareness). This is because the meaning of social presence adopted in this paper is reflected better in these last two indicators. The final version of the questionnaire attempted to encapsulate the social presence perceived through the interface. In addition, items in the last version of the questionnaire were reworded, and text was substituted for the Likertscale numbering, facilitating better comprehension of the scale. Therefore the final version of the social presence questionnaire is short and composed of clear and concise items.

\section{Acknowledgements}

First and foremost, I would like to thank Mary C. Dyson for always helping me towards clarity when I needed it most. I would also like to acknowledge that the research described in this paper was made possible by my sponsor CNPq, Brazil.

\section{References}

[1] Biocca, F., Burgoon, J., Harm, C., \& Stoner, M. (2001). Criteria and scope conditions for a theory and measure of social presence Available: http://www.temple.edu/ispr/prev_conferences/proceedings/ 2001/Biocca1.pdf [2007, 29 March ].

[2] Breakwell, G. M., Hammond, S., \& Fife-Schaw, C. Research methods in psychology (2 ed.). Sage Publications, London, 2000.
[3] Cook, S. Ethical implications. In L. H. Kidder (Ed.), Research methods in social relations (4th ed.). Holt, Rinehart \& Winston, New York, 1981, pp. 365-417.

[4] Fadel, L. M., \& Dyson, M. C. “A Comparison of the perception of social information in text and visual-based interfaces”. 12th International Conference on Technology Supported Learning \& Training - Online Educa Berlin, Berlin, 2006.

[5] Garrison, D. R., Anderson, T., \& Archer, W. "Critical inquiry in a text-based environment: Computer conferencing in higher education". Unpublished manuscript, Edmonton, 2000.

[6] Gunawardena, C. N., \& Zittle, F. J. (1997). Social presence as a predictor of satisfaction within a computermediated conferencing environment. The American Journal of Distance Education, 11(3), 8-26.

[7] Howell, D. C. Statistical methods for psychology (5 ed.). Duxbury, Pacific Grove, 2002.

[8] Rourke, L., Anderson, T., Garrison, R., \& Archer, W. (2001). Assessing social presence in asynchronous textbased computer conferencing. Journal of Distance Education/Revue de l'enseignement à distance, 14(2), 1-18.

[9] Short, J., Williams, E., \& Christie, B. (1976). The social psychology of telecommunications. London: John Wiley \& Sons.

[10] Tu, C.-H. (2002). The impacts of text-based CMC on online social presence. Journal of Interactive Online Learning, 1(2), 1-24. 